\title{
Simulating a Multi-Vehicle Traffic Sensing System Based on Radio Tomographic Imaging
}

\author{
Jarmo T. Wilkens, Jair A. Silva, Anilton S. Garcia \\ Laboratory of Telecommunications (LabTel), Department of Electrical Engineering, Federal University of \\ Espírito Santo (UFES), Av. Fernando Ferrari, 514, Campus Goiabeiras, Vitória-ES, Brazil \\ jarmowilkens@gmail.com, anilton.garcia@ufes.br,jair.silva@ufes.br
}

\begin{abstract}
One of the most recently developed wireless technologies is Radio Tomographic Imaging (RTI). RTI employs a wireless sensor network that produces images of the change in the electromagnetic field of a monitored area using Received Signal Strength (RSS) measurements. This allows the tracking of devicefree objects such as humans and cars. This paper is the first to propose and validate a simulation model that simulates RSS measurements for arbitrary RTI networks, based on the ZigBee communication protocol. The simulation model allows the specification of an RTI network from the ground up, including node positions, network size and geometry and RSS measurement processing. Furthermore, this paper demonstrates the implementation of the simulation model to an enhancement of a recently proposed RTI system, which acts as a roadside surveillance system. The enhancement includes three newly proposed techniques, namely a new weight matrix calculation method, a new node spacing setup and a new vehicle detection method. The simulation results indicate that it is possible to detect both one or two family sized cars simultaneously. Using techniques that reduce RSS variance due to multipath effects and the newly proposed methods, simulated vehicle detection performance is demonstrated to be between $95 \%$ and $100 \%$.
\end{abstract}

Index Terms - Radio Tomographic Imaging, Received Signal Strength, Simulating Wireless Sensor Networks, Vehicle Traffic

\section{INTRODUCTION}

One of the most recently developed and promising wireless technologies is Radio Tomographic Imaging (RTI). RTI is a technique that generates an image of the electromagnetic field of a measured area. Using a mesh network of radios positioned on the borders of the measured area, RTI enables the localization and tracking of device-free targets. Objects inside the network attenuate the $2.4 \mathrm{GHz}$ ISM band signals and this attenuation can be measured by obtaining the Received Signal Strength (RSS) measurements of each link. Retrieved RSS data is used to generate an RTI image, where blobs inside the image represent the location estimate of a target. Initially developed in 2008 by Patwari et al. [1], RTI is an emerging and promising technology. Recent relevant RTI research were presented by [2], [3]. 
Simulating RSS measurements for each link in an RTI system has an important benefit, as it allows an arbitrary network geometry and size to be evaluated before buying and installing components. This paper is the first to propose a simulation model that simulates RSS measurements based on a simulated communication channel in an arbitrary RTI network setup.

Currently, no publications of a proposed simulation model of an RTI system exist. Only two RTI simulation models exist in literature, which are both proposed in dissertations by Cooke [4] and Milburn [5]. The simulated RSS data in both cases is reverse engineered using an initial semirandomly generated image and mathematical RTI models.

The main limitation of their simulation models is that they lose accuracy of the simulated RSS data during the process due to their approach of reverse engineering the RSS data. The advantage of the proposed simulation model over these previous models is that it implements physical layer properties by simulating a communication channel based on the ZigBee protocol. This makes it possible to include multipath components and a Rician channel, which both contribute to make the simulated RSS data more realistic and accurate. Furthermore, it implements the link-channel pair selection method [3] that reduces multipath effects and RSS behavioral characteristics described in literature to obtain a more realistic and accurate simulation model. The proposed simulation model allows the specification of an RTI network from the ground up, including node positions, network size and geometry, as well as RSS measurement processing using any available or newly developed techniques. The model is relatively flexible in that it can be used for different types of network geometries, such as a square geometry as is used in previous works, as well as a front-back geometry like the one discussed in this paper.

In addition to proposing a new simulation model, this paper presents the implementation of the simulation model to an enhancement of the recently proposed RTI system by Wilkens et al. [6]. This RTI system proposes the estimation of the number and velocity of multiple vehicles on a single lane road segment with radios deployed on the roadside that form an RTI network. The enhancement includes three newly proposed techniques, namely a new node spacing approach, a new weight matrix calculation (WMC) method and a new pixel-specific threshold calculation technique for vehicle detection.

The rest of this paper is organized as follows. Section II discusses the new enhancements concerning the recently proposed RTI system by Wilkens et al. [6]. Section III describes the proposed simulation model with validation and implementation of the RTI system. The results after implementation of the proposed RTI system are presented in section IV and section V concludes this article.

\section{PROPOSED RTI SYSTEM}

The proposed RTI system that is simulated is an extension of the RTI system proposed by Wilkens et al. [6]. Instead of deploying evenly spaced nodes along the roadside, non-linear node spacing was 
chosen to increase link density, and thereby performance, at the sub-network edges. Fig. 1 illustrates the proposed design as a top view, where the blue circles represent the nodes. Three sub-networks are displayed, which are justified by Wilkens et al. [6].

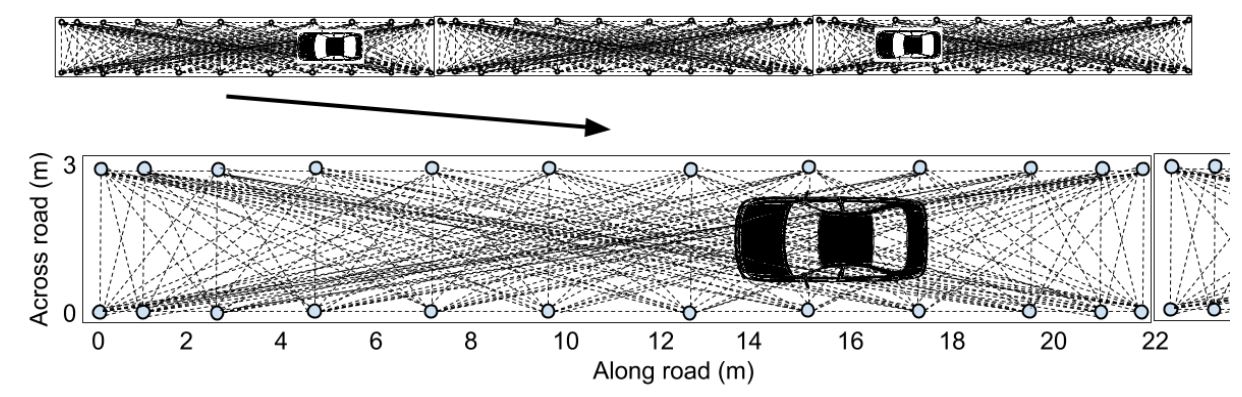

Fig. 1. Top view of roadside surveillance proposal

Network scan frequency is dependent on node transmission time and number of channels used. A trade-off exists between system performance and maximum detectable velocity, where improving localization accuracy would require using a higher amount of frequency channels due to more reliable link-channel pairs being selected, which comes at the cost of network scan frequency and thus maximum detectable velocity. The system proposed by Wilkens et al. [6] has a network scan frequency of $7 \mathrm{~Hz}$, employs two frequency channels and has a pixel size of $2 \times 2 \mathrm{~m}^{2}$. Fig. 9(a) visualizes the location of the pixels as blue squares in an arbitrary sub-network.

\section{A. Radio Tomographic Imaging Algorithm}

The enhanced algorithm employs the same methods as the recently proposed RTI algorithm [6], except that it uses a different WMC method, a node-specific path loss model and a new, but similar pixel-specific threshold technique.

During initialization, the node and pixel locations need to be obtained in order to calculate the weight matrix $\mathbf{W}$. This weight matrix indicates the extent to which each link represents each pixel. Node locations are as shown in Fig. 1. The new WMC method is based on placing a circle with radius $0.7 \mathrm{~m}$ at the center of each pixel, which is illustrated in Fig. 9(b). All links that pass through the circle are selected. For the network setup proposed in this paper, this method selects more useful links for each pixel and thereby leads to improved system performance, as demonstrated in subsection IV.B.1).

Fade level indicates the reliability of a link-channel pair. As demonstrated by Bocca et al. [2], a higher (lower) fade level generally leads to less (more) RSS link variance. Positive (negative) fade level links are referred to as anti-fade (deep fade) links, as a result of constructive (destructive) multipath interference at the receiver. Fade level is calculated using the path loss model and reference RSS vector. The reference RSS vector is obtained during offline calibration, which is where the system records RSS data without targets inside the network. To increase accuracy of the fade level, the path loss model parameters are node specific, as presented by Alippi et al. [3]. In other words, each node $n$ is modeled to have its own path loss exponent $\eta_{n}$ and transmit reference power $P_{0}^{n}$. Alippi et al. [3] mention that using a node-specific path loss model, the system is more robust against 
hardware variability factors (including relative antenna orientation between communicating radios) and local environmental differences (like proximity of nodes to dense foliage). The link-channel pair selection method proposed by Alippi et al. [3] will select the highest quality anti-fade link-channel pairs that experience least RSS link variance. This filters out unreliable link-channel pairs that experience deep fading, as well as it filters out the longer distance links that have an average RSS close to the sensitivity threshold.

The system outputs are the number of detected cars, car location and speed. Every pixel that has an intensity higher than the pixel-specific threshold is considered to be occupied by a vehicle. This threshold is new, but similar to the pixel-specific threshold $\mathcal{T}_{v}$ that was presented by Wilkens et al. [6] given by:

$$
\mathcal{T}_{\mathrm{V}}=\rho\left(\sum_{\mathrm{k} \in \mathcal{A}_{v} .} \mathrm{F}_{\mathrm{k}}\right)^{\frac{1}{\mathrm{n}}}
$$

where $\rho$ and $n$ are empirically determined parameters, $\mathcal{A}_{v}$ is the set of selected link-channel pairs $k$ covering pixel $v$ and $F_{k}$ is the fade level. The basis of this threshold depends on the fact that pixel intensities in general are lower on network edges than in the network center, due to differences in link densities respectively, where more (less) RSS information is available in regions with higher (lower) link densities. Differences in link density along the RTI network can be observed in Fig. 1. Therefore, the threshold is dependent on amount of selected link-channel pairs given by $\mathcal{A}_{v}$. A lower threshold is thus assigned to the border pixels, whereas the center pixels will be given a higher threshold. Furthermore, the threshold is dependent on fade level, because links with a higher (lower) fade level lead to a higher (lower) pixel intensity, because they experience more (less) signal attenuation. Parameters $\rho$ and $n$ shape the threshold curve and are optimized to obtain optimized system performance. If there are multiple neighboring pixels detected as a car, only the pixel representing the front part of the car is chosen, so the direction of traffic will need to be known a priori. Vehicle speed is estimated as the number of pixels displaced per second, multiplied by the pixel width.

The proposed algorithm includes online calibration at the start of each network scan, which helps reduce RSS time-variance due to random objects being placed inside the network, or weather effects.

\section{Simulation MODEL}

The simulation model is implemented in MATLAB and is based on experimental (raw) data [7] that Wilson and Patwari published online and which was used in their published article [8]. The researchers used the Texas Instruments (TI) CC2531 transceiver that communicates through the ZigBee protocol [9] in the $2.4 \mathrm{GHz}$ ISM band, so the simulation model is based on the ZigBee protocol (which relies on the IEEE 802.15.4 standard) and the transceiver's properties. The simulation model is also based on the RSS dynamics outlined by Bocca et al. [2]. Furthermore, this section 
shows how the simulation model can be utilized to implement and evaluate the performance of the proposed RTI system.

The initialization of the simulation model consists of setting node and pixel locations, calculating link distances, the weight matrix and regularization matrix. The regularization matrix is further explained in subsection III.B.5). The simulation model is divided in two sections: subsection III.A covers the procedure to obtain RSS data, whereas subsection III.B outlines how RSS data is processed and used to generate new information until a Radio Tomographic Image estimate is obtained. The procedures detailed in III.A and III.B.1) - III.B.3) only need to be computed once, while III.B.4) III.B.6) are repeated for every network scan. The rest of this section demonstrates the model validation.

\section{A. Obtaining RSS data}

The procedure of obtaining RSS data is mainly based on the convolution between a communication channel, which is modeled as a Rician channel, and a transmitted data signal generated by a simulated ZigBee protocol for the 2.4 GHz ISM band defined by the IEEE 802.15.4 standard [9]. The output of the convolution between the transmitted signal (III.A.1) and the Rician channel (III.A.2) with AWGN is used to obtain the RSS measurements (III.A.3) for each link.

The procedures detailed in this section are repeated for each used frequency channel. In the simulation model, only the number of frequency channels to be used is specified, rather than the defining the specific frequency channel.

\section{1) Transmitted signal}

Generating a transmitted data signal consists of four stages.

1. The first is to obtain a data frame. The data frame contains the preamble (4 bytes), start frame delimiter (SFD) (1 byte), frame length field and a reserved bit (total 1 byte) and the data unit. The data unit contains 2 bytes for the node ID and 1 byte per RSS measured from each node (so $N-1$ bytes, where $N$ is number of nodes). Therefore, a random (uniformly distributed) binary data sequence of $(7+N) \times 8$ bits is generated.

2. The second stage consists of implementing the Direct Sequence Spread Spectrum (DSSS), by converting the bits of stage 1 to 4-bit symbols. Afterwards, the symbols are mapped into a 32-chip pseudorandom (PN) sequence. As the data rate of the original signal is defined to be $250 \mathrm{~kb} / \mathrm{s}$ [10], the over-the-air bandwidth after spreading becomes $2 \mathrm{MHz}$.

3. The third stage modulates the chip sequence with the Offset Quadrature Phase Shift Keying (OQPSK) modulation scheme.

4. Stage 4 consists of filtering the Q- and I-Phase signals using the Half-Sine pulse shaping filter. 


\section{2) Communication Channel}

To model the communication channel, a Rician channel was used, because most of the communication links are assumed to be Line-of-Sight (LOS), whereas a Rayleigh channel would be used for non-LOS cases [11].

The number of multipaths is related to the signal bandwidth and coherence bandwidth. Since the coherence bandwidth is not known a priori, the number of received paths per signal for this simulation model was chosen to be 3 . Therefore, the vectors representing path delays and path gains have three elements. The first path delay refers to the first arriving path and is set to zero [12]. The subsequent path delays were set to be random (uniformly distributed) multiples of the sample time of $62.5 \mathrm{e}-9 \mathrm{~s}$, where the second path always arrives earlier than the third path.

The path gains are related to the log-distance path loss model parameters found for the "empty area" data published by Wilson et al. [7]. The parameters found were $P_{0}=-52.82 \mathrm{dBm}$ at $d_{0}=1 \mathrm{~m}$ and $\eta=1.57$. Around half of all links were simulated to be with positive fade level, which is according to the data of Wilson et al. [7]. For every link, an initial path gain for the first path was calculated using the log-distance path-loss model. If that link was detected to be reliable, a gain offset of $5 \mathrm{~dB}$ was added to the initial path gain to give it positive fade level (anti-fade), whereas for unreliable links 5 $\mathrm{dB}$ was subtracted from the initial path gain. See section III.C about why a $5 \mathrm{~dB}$ gain offset was chosen. For the subsequent path gains, $2 \mathrm{~dB}$ was subtracted from the preceding path gain and a random (uniformly distributed) decimal value in the interval [-1,1] was added to it. The Rician channel was then filtered with the transmitted signal. Fig. 5(a) shows the simulated RSS vs. logdistance and its log-distance path loss model with the same network setup as Wilson et al. [8].

\section{3) Received Signal Strength Measurements}

The Received Signal Strength Intensity (RSSI) $\bar{r}_{l, c}$ value is obtained by averaging the received power over the first eight symbols following the SFD directly after the demodulator [9], [10]. Since in the simulation model the received signal is discrete and shifting from an intermediate frequency to baseband is not applicable, the RSSI was calculated directly from the received signal without preprocessing.

\section{B. Processing RSS data}

The steps from processing RSS data until a Radio Tomographic Image estimate include computing fade level, selecting link-channel pairs, calculating thresholds and simulating a vehicle.

\section{1) Fade level}

Fade level is dependent on the log-distance path loss model as explained in section II.A. The path loss parameters $\eta$ and $P_{0}$ were calculated by finding the linear model when plotting all simulated RSS data against the logarithm of corresponding link lengths. Fig. 5(b) shows the fade level of the simulated RSS with the same network setup as Wilson et al. [7]. 


\section{2) Link-channel pair selection}

The link-channel pair selection method was first presented by Alippi et al. [3] and contains four stages.

1. The set of link-channel pairs with positive fade level was obtained.

2. A second set of link-channel pairs was retrieved that contained RSS measurements higher than the grey region (receiver sensitivity threshold).

3. The intersection between the previously found sets was obtained.

4. The fourth stage is dependent on RSS variance $\sigma_{l, c}^{2}$ of link $l$ on channel $c$ to calculate the weights, many realizations would have to be computed to calculate such variance. As each realization takes about 23 seconds to compute, a different solution was found to obtain RSS variance to reduce simulation time. The experimental data of Wilson et al. [7] was used to find a relationship between fade level and variance. This relationship is displayed in Fig. 2. As can be observed, two curves were found as a best fit that describe the relationship between variance of links and channels experiencing negative and positive fade levels. These curves were found by ignoring some of the data in the higher variance levels on the positive fade level side. This data was regarded as outliers, because they do not explain the behavior as described by Bocca et al. [2]. Curve (1) in the negative fade level section corresponds to $\sigma_{l, c}^{2}\left(\mathrm{dBm}^{2}\right)=1.5-0.25 F_{l, c}$ and curve (2) describes variance of links and channels in positive fade level by $\sigma_{l, c}^{2}\left(\mathrm{dBm}^{2}\right)=1.5-0.05 F_{l, c}$.

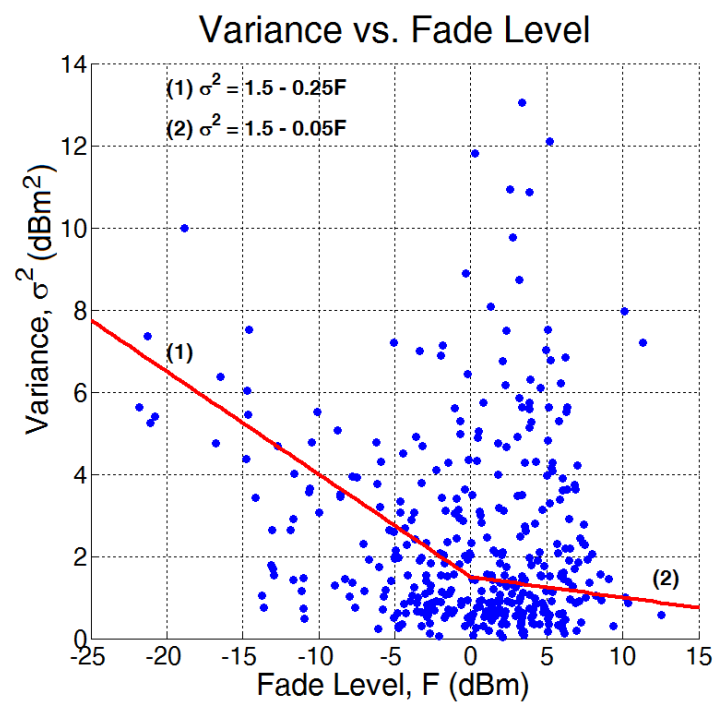

Fig. 2: RSS variance as a function of fade level. The displayed data was processed from experimental data of Wilson et al. in the empty area. Curves (1) and (2) in the negative and positive fade level sections correspond to the formulae displayed in the top left side of the graph.

\section{3) Threshold}

The threshold $\mathcal{A}_{v}$ was calculated as given by Equation (1). Parameters $\rho$ and $n$ were found empirically to yield the best performance and are given in subsection IV.B.4). 


\section{4) Vehicle simulation}

The procedure described in this subsection contains three stages and was repeated for every network scan, as it changes the RSS data according to the behavior described by Bocca et al. [2].

1. In the first stage of the simulation model, a vehicle is assumed to be four meters in length, which corresponds to the length of two neighboring pixels that are a size $2 \times 2 \mathrm{~m}^{2}$ each. All the links chosen during the weight matrix calculation that represent the pixels occupied by a vehicle are found.

2. All the links in the network are assigned a RSS standard deviation $\sigma_{l, c}$ according to the Equations presented in III.B.2). A new RSS measurement $r_{l, c}$ is calculated for each link on each channel by:

$$
\mathrm{r}_{1, \mathrm{c}}=\overline{\mathrm{r}}_{1, \mathrm{c}}+\sigma_{1, \mathrm{c}} \times \operatorname{randn}
$$

where $\bar{r}_{l, c}$ is the RSS data calculated in III.A.3) and randn is a MATLAB function that generates a zero mean unit variance random Gaussian variable. This represents the RSS noise, which is assumed AWGN by Anderson et al. [13].

3. The third stage is where the vehicle is simulated. As shown in an experiment by Bocca et al. [2], when a target is present on the RSS link in anti-fade, the RSS drops with around $5 \mathrm{~dB}$, while RSS links in deep fade do not tend to be affected by it due to their high RSS variance. Unfortunately, this is the only link blocking behavior knowledge available, meaning it was not possible to develop or use an already existing model to determine the signal attenuation of an arbitrary object size that blocks links of arbitrary length and fade level. Therefore, a fixed signal attenuation value was used to subtract from $r_{l, c}$ for every anti-fade link-channel pair that is part of the set found in stage 1 of the described vehicle simulation procedure. The fixed signal attenuation value is set to $8 \mathrm{~dB}$, as it is assumed that vehicles attenuate signals more than humans do (around $5 \mathrm{~dB}$ ), but not significantly more.

\section{5) Obtaining a Radio Tomographic Image estimate}

After the RSS vector is updated $r_{l, c}$, the change in RSS vector $y_{l, c}$ is calculated by $y_{l, c}=\bar{r}_{l, c}-r_{l, c}$. All the elements in $y_{l, c}$ that do not belong to the set of selected link-channel pairs are set to zero.

The radio tomographic image estimate vector $\hat{\mathbf{x}}$ is computed by:

$$
\widehat{\mathbf{x}}=\Pi \mathbf{y}
$$

where regularization matrix $\Pi$ may contain a Tikhonov or covariance matrix [8], [2], where the former is a more generalized solution and the latter is more specific. For the simulation of the proposed RTI system, it was chosen to employ general regularization, given by:

$$
\Pi=\left(\mathbf{W}^{\mathrm{T}} \mathbf{W}+\alpha\left(\mathbf{D}_{\mathrm{X}}^{\mathrm{T}} \mathbf{D}_{\mathrm{X}}+\mathbf{D}_{\mathrm{Y}}^{\mathrm{T}} \mathbf{D}_{\mathrm{Y}}\right)\right)^{-1}
$$

where the difference matrices $\mathbf{D}_{\mathrm{X}}$ and $\mathbf{D}_{\mathrm{Y}}$ are the difference operators for the horizontal and vertical directions of a 2D image respectively (see [4] for a detailed example). Anderson et al. [13] show that smoothing parameter $\alpha$ can be set to $\alpha \approx 0.3$ for weak smoothing and $\alpha \approx 30$ for strong smoothing. 
Removing negative observations in RTI image $\mathbf{x}$ as proposed by Anderson et al. [13] was implemented to reduce image noise. This procedure was iterated until the RTI image did not contain negative pixel intensities anymore, which was never more than one or two iterations.

\section{6) Vehicle detection and speed estimation}

The vehicle detection method was implemented exactly as explained in section II.

\section{Simulation Validation}

To validate the simulation model, all of the data of all 378 links of the experimental RSS data was combined giving 100,992 measurements. All the RSS data (in voltage) was then shown in one probability distribution. The application "dfittool" in MATLAB was used to find a distribution that most closely fits the histogram generated from the RSS data, where the number of bins used was 32 . A distribution was chosen manually and the distribution with highest log likelihood value (calculated by the application) representing the histogram of the experimental RSS data was selected to represent the histogram of both experimental and simulated RSS data. It was found that the Burr Type XII distribution most closely fits the histogram of the experimental RSS data, which are both shown in Fig. 3.

The next stage in validation was to generate 20 realizations of simulated RSS data, where each realization contained 100,926 RSS elements. One realization consisted of obtaining one RSS measurement for each of the 378 links using the method described in section III.A. The rest of the measurements were simulated by repeating the procedure of the second stage of subsection III.B.4). All RSS data was converted from $\mathrm{dBm}$ to voltage and for each realization, a respective Burr probability distribution was calculated, which were then averaged to obtain a more generalized result that is less prone to variations caused by the Rician channel object. The resulting distribution was validated to the probability distribution of the experimental RSS data according to the Mean Square Error (MSE) metric of the probability values.

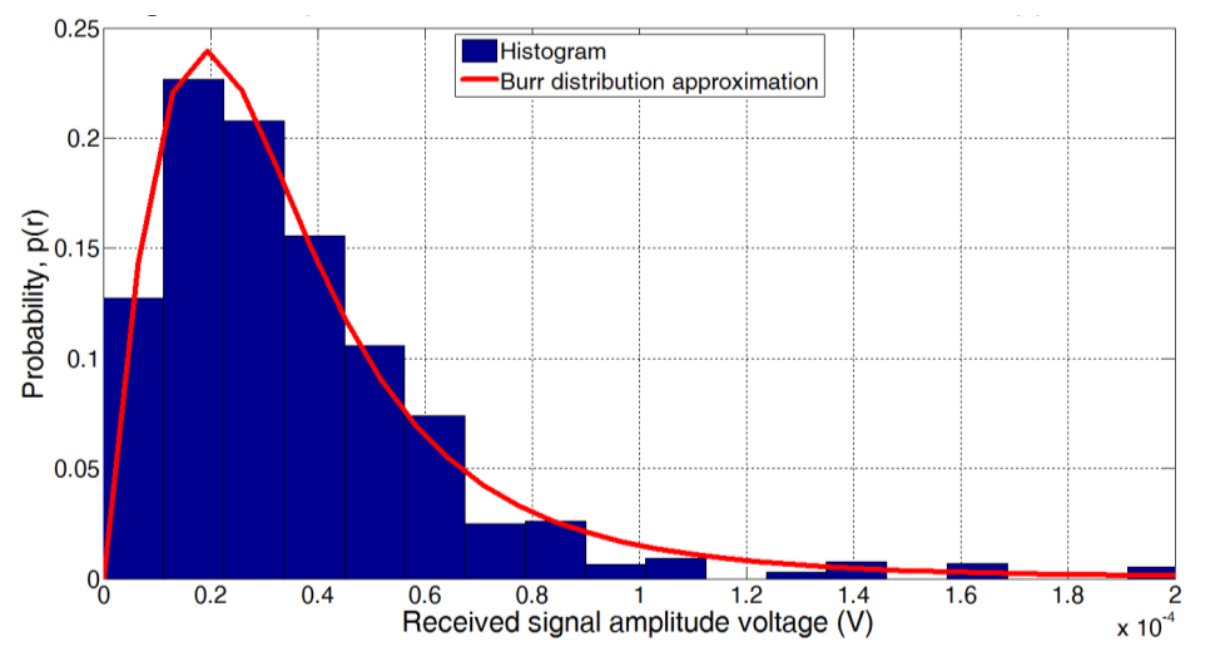

Fig. 3: Histogram of experimental RSS data with its Burr distribution approximation. 
This validation procedure was repeated for four different values of three simulation parameters to optimize the fit of the simulation distribution to the experimental distribution. The parameters that were optimized were the path-loss model parameters $P_{0}, \eta$ and the gain offset, which all affect the simulated path gains described and hence the shape of the respective RSS probability distribution. Table 1 presents the MSE values of the three parameters. Fig. 4 presents the optimized simulation distribution with a MSE of $0.17 \times 10^{-4}$ using parameter values $P_{0}=-50.82 \mathrm{dBm}, \eta=1.37$ and gain offset $=5 \mathrm{~dB}$. Observe that parameter $P_{0}$ is the same as obtained from the experimental data, but parameter $\eta$ was reduced from 1.57 to 1.37 . Note that the optimized result presented in this paper is likely not the best possible result, since only the described parameter values were evaluated. As it took around 30 minutes to generate one graph, it was left outside the scope of this research to find the best possible result.

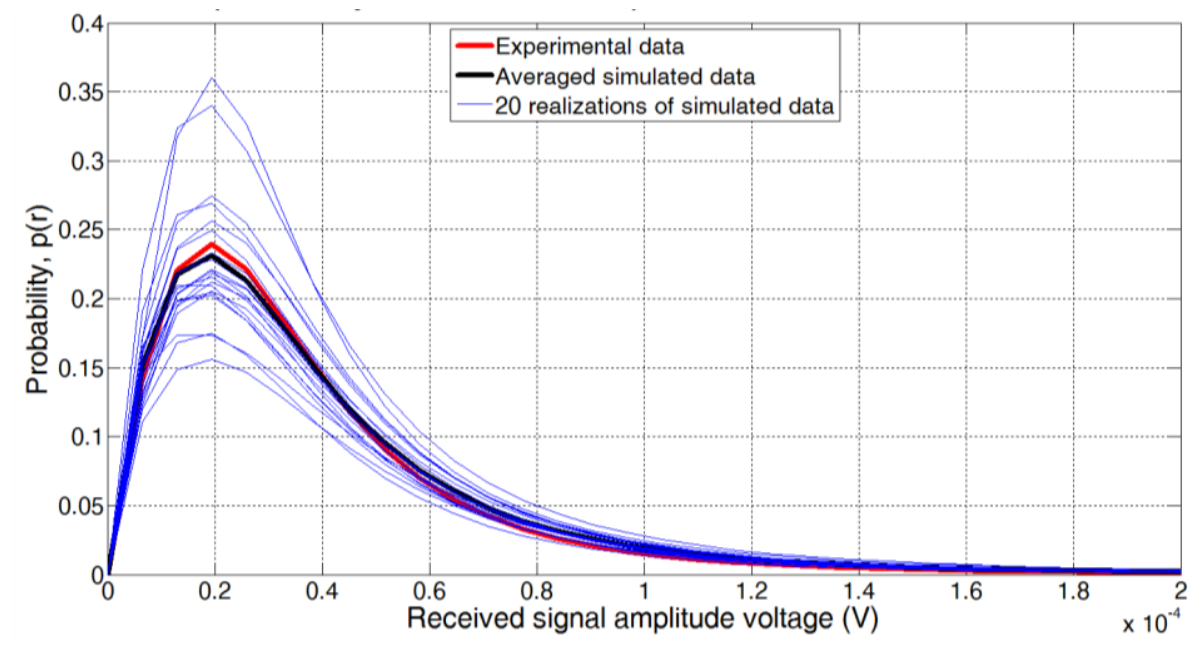

Fig. 4: Burr probability distribution approximation of histogram of experimental RSS data (red line) from Wilson et al. [7] and simulated data, with path-loss parameters $P_{0}=-50.82 \mathrm{dBm}, \eta=1.37$ and gain offset $=5 \mathrm{~dB}$. MSE $=0.17 \times 10^{-4}$

Table 1: Table comparing MSE between Burr probability distribution approximation of histogram of experimental RSS data and averaged simulated RSS data with different values for $P_{0}, \eta$ and gain offset

\begin{tabular}{cccccc}
\hline $\boldsymbol{\eta}$ & $\mathbf{M S E}\left(\times \mathbf{1 0}^{-\mathbf{4}}\right)$ & $\left.\boldsymbol{P}_{\mathbf{0}} \mathbf{( d B m}\right)$ & MSE $\left(\times \mathbf{1 0}^{-\mathbf{4}}\right)$ & Gain offset $(\mathbf{d B})$ & MSE $\left(\times \mathbf{1 0}^{\mathbf{- 4}}\right)$ \\
\hline 1.17 & 5.65 & -48.82 & 5.18 & 1 & 7.07 \\
1.37 & 1.85 & -49.82 & 2.46 & 3 & 1.82 \\
1.57 & 4.01 & -50.82 & 1.27 & 5 & 0.32 \\
1.77 & 11.00 & -51.82 & 4.36 & 7 & 5.48
\end{tabular}

Fig. 5(a) and Fig. 5(b) show the simulated RSS vs. log-distance and fade level respectively with the optimized parameters representing the same network setup as Wilson et al. [8]. As can be observed, parameters $P_{0}$ and $\eta$ are not exactly the same as the optimized parameters, due to the partial randomness of the Rician channel. Furthermore, the maximum and minimum fade levels approximately correspond to the experimental fade levels (not presented in this paper). 
(a) Simulated RSS vs. Log-Distance

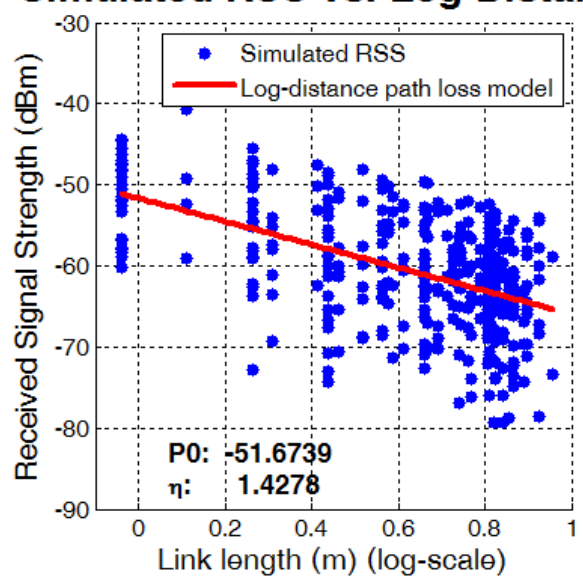

(b)

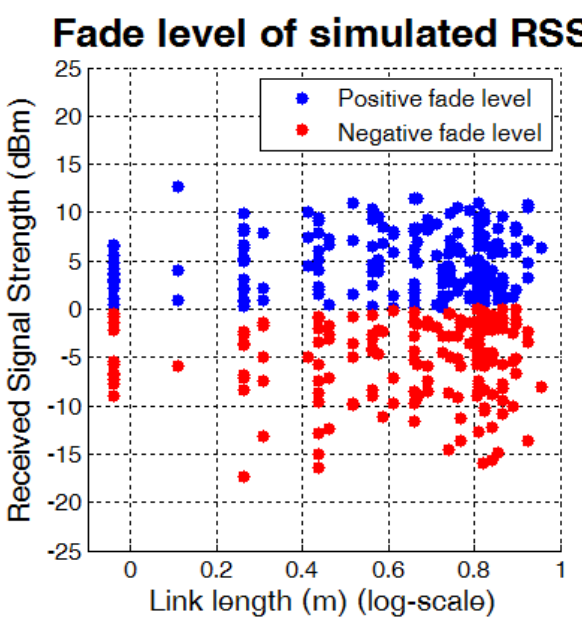

Fig. 5: Fig. (a) shows simulated RSS (dBm) data versus log-distance $(\mathrm{m})$ and its log-distance path-loss model with corresponding parameters $P_{0}$ and $\eta$ displayed in the graph. Fig. (b) shows the fade level, where the blue and red data points are the positive and negative fade levels respectively, shown to more easily distinguish between the two levels. 


\section{RESULTS}

The results presented in this section demonstrate the implementation of the proposed RTI network described in section II using the simulation model outlined in section III. In section IV.A, the optimized result is shown for the implementation of one and two vehicles. How this result was optimized is covered in section IV.B.

\section{A. Optimized result}

This section shows the simulation results of one and two vehicles present inside an arbitrary subnetwork in subsections IV.A.1) and IV.A.2) respectively.

1) Single vehicle

Simulation results of a single family sized car of 4 meters located on each pixel from 1 to 11 in an arbitrary sub-network is shown in Fig. 6 and Fig. 7, displayed as a line plot and image respectively.

As can be observed in Fig. 6, using the validated simulation model, a car passing through an RTI network as a roadside surveillance application can clearly be located. A vehicle is detected on any pixel with pixel intensity higher than the threshold, which, purely by observation, is accurate in each of 11 cases. The pixel intensities of the car located on the edge of the network is roughly speaking lower than when it is located in the network's center. This is due to link density, which is lower on the edge than in the center and the threshold value is adjusted to this characteristic accordingly, showing a higher value in the middle than on the sides.

Another observation is that the image is still minimally affected by noise, due to RSS link variance and multipath effects, as seen on the pixels further away from the current vehicle location. Most of the noise has been filtered out by using the link-channel pair selection method, which is demonstrated in subsection IV.B.3). The simulation model therefore does not show an ideal case with zero noise, but rather a more realistic representation of a practical implementation where noise is minimized.

Fig. 7 shows the RTI image version of Fig. 6, where the two brightest neighboring pixels in each image (a) to (k) represent the location of a vehicle. The pixel intensities are a result of a reconstruction algorithm given by Equation (3) that uses noisy RSS measurements of selected link-channel pairs. This is the final output of the RTI technology. 
(a)

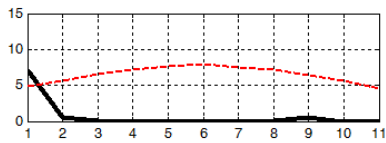

(c)

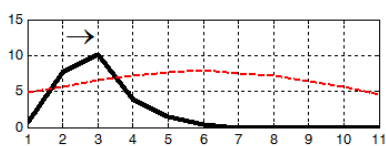

(e)

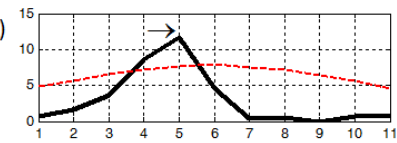

(g)

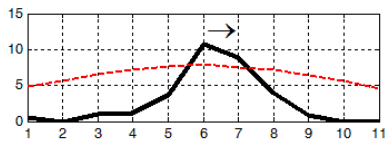

(i)

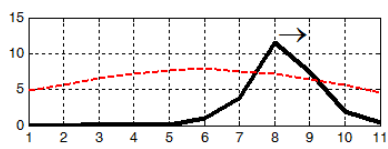

(b)

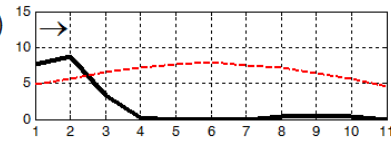

(d)

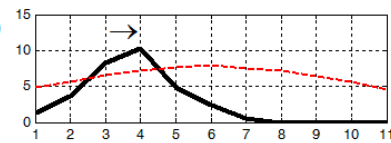

(f)

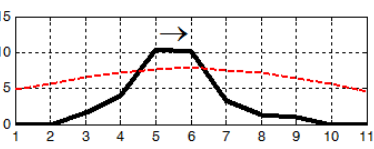

(h)

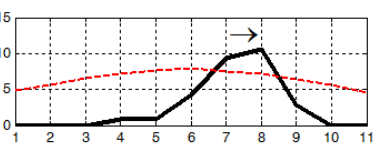

(j)

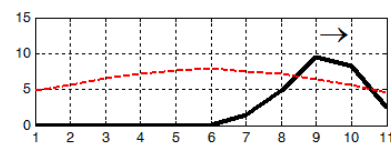

(k)

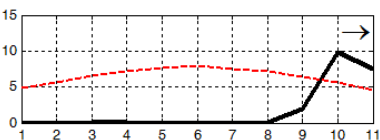

Fig. 6: Simulated car (black curve) on pixels 1 to 11 (x-axis) shown in Figs. (a) to (k) respectively, where the y-axis represents pixel intensity. The red dashed curve is the pixel-specific threshold and the arrow indicates the two respective pixels occupied by the car and the flow direction.

(a)

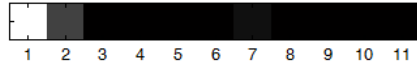

(c)

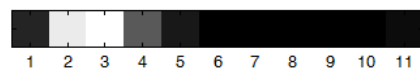

(e)

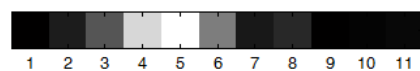

(g)

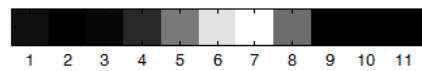

(i)

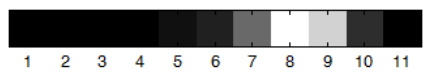

(b)

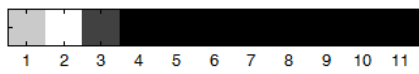

(d)

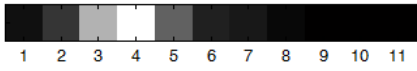

(f)

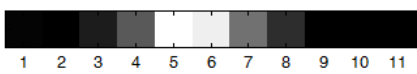

(h)

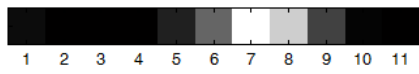

(j)

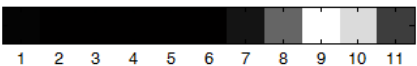

(k)

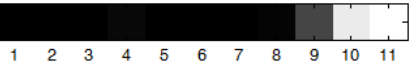

Fig. 7: Simulated car shown as the two brightest neighboring pixels in each image on pixels 1 to 11 shown in Figs. (a) to (k) respectively. This is the RTI image representation of Fig. 6

2) Two vehicles

This paper proposes the detection and localization of multiple vehicles using the RTI network setup described in section II. The implementation of this proposal with two vehicles is simulated and presented in Fig. 8.

The RTI image with two vehicles clearly shows two vehicles located on pixels 2 and 3 , and 7 and 8 respectively. The line plot also indicates that the highest intensities are measured on the mentioned pixels and are all higher than the threshold. This means that in theory, according to the simulation model and the assumptions made in it, the system would successfully detect the location of both vehicles in the given scenario. However, system performance is expected to be lower compared to one vehicle, because some links are blocked by two vehicles at the same time, thereby reducing the reliability of the respective links. 
(a)
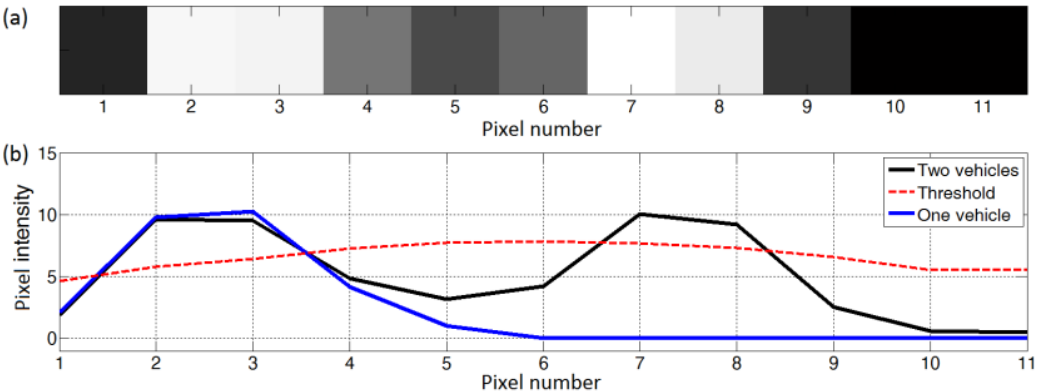

Fig. 8: RTI image with two vehicles located on pixels $[2,3]$ and $[7,8]$ respectively and its corresponding representation as a line plot (black curve). The effect of two vehicles in the network can be compared to the effect that one vehicle has by the blue curve. The red dashed curve is the threshold.

This phenomenon of links being blocked by two vehicles can be observed in Fig. 8(b). The intensity on pixel 5 is higher with two vehicles than one vehicle (blue curve). This is due to the fact that the links representing this pixel pass through both vehicles, thereby experiencing more signal attenuation and hence resulting in a higher pixel intensity than if those links would pass through only one vehicle. This phenomenon in a practical situation is thus also reflected in the simulation model.

\section{B. Optimizing the result}

The results presented in section IV.A are optimized with respect to two newly proposed techniques and one existing technique. The parameters used for the optimized result are summarized in IV.B.4).

\section{1) New Weight Matrix Calculation and its effect}

One of the newly proposed techniques is the link selection procedure for the weight matrix. The original method [8] consists of using an ellipse that represents the area measured by one link, where the larger the ellipse width, the higher the chance that it covers more pixels (dependent on pixel size). This is a logical and effective method for 2-dimensional images with a relatively high pixel density.

For this proposal, however, a 1-dimensional image is used with relatively few pixels of a large size $\left(2 \times 2 \mathrm{~m}^{2}\right)$. The reason why this method is more effective for the given situation, is that it allows the more valuable links to be chosen, and thus it prevents the selection of links that provide relatively little information about a given pixel. This effect is shown in Fig. 9, where both methods are compared to each other. The selected links on pixels 1 and 10 are displayed by the red links, the unselected links are shown as gray lines, the pixels are shown as blue squares and the selection circle is dark green. The number of selected links is shown on top of the corresponding pixel.

As can be observed, using the original method, four links are selected for the first pixel and 16 for the tenth pixel. The new method selects 4 and 12 links for the first and tenth pixel respectively. The four extra links selected by the original method for pixel 10 are those that provide relatively little information about that pixel, as they are longer links and pass through more pixels. Reducing the ellipse width would remove those links. However, this would also remove two of the longer links of the first pixel, which is undesired, because those to-be-removed links pass almost half of their length through that pixel, meaning they are valuable links that represent that pixel. Alternatively, one may want to use only the four nodes around each pixel with at most four links to determine its intensity, 
instead of making use of links that pass through several pixels that just add noise and complexity to the reconstruction algorithm. In a practical situation, however, it may occur that one or more of these four links experience deep fade (very unreliable links). Therefore, using only four nodes per pixel, and filtering out deep fade links, few or maybe no links (in the worst case) may be used to measure that pixel, thereby making that pixel more inaccurate and vulnerable. See Fig. 5 to notice that short links are filtered out due to them experiencing deep fade. Hence, as also used in previous RTI works, it is more desirable to combine information of as much selected link-channel pairs as possible to measure a pixel. The increased noise and unreliability of links that cover multiple pixels is taken into account in the more complex weight matrix: shorter links (which cross fewer pixels) are given a larger weight than longer links.

Vehicle detection performance between the two selection methods is presented in Fig. 10. Performance was measured by calculating the average detection accuracy $(\%)$ of 50 repetitions on pixels 1 to 11 . The output of the performance computation is a 1 or 0 for each repetition, where a 1 is that the location of the front half of the car is correctly identified and 0 otherwise. In each repetition, the RSS vector is updated by the procedure described in subsection III.B.4). This process is one simulation realization, which was repeated 20 times and averaged to obtain a more general result.
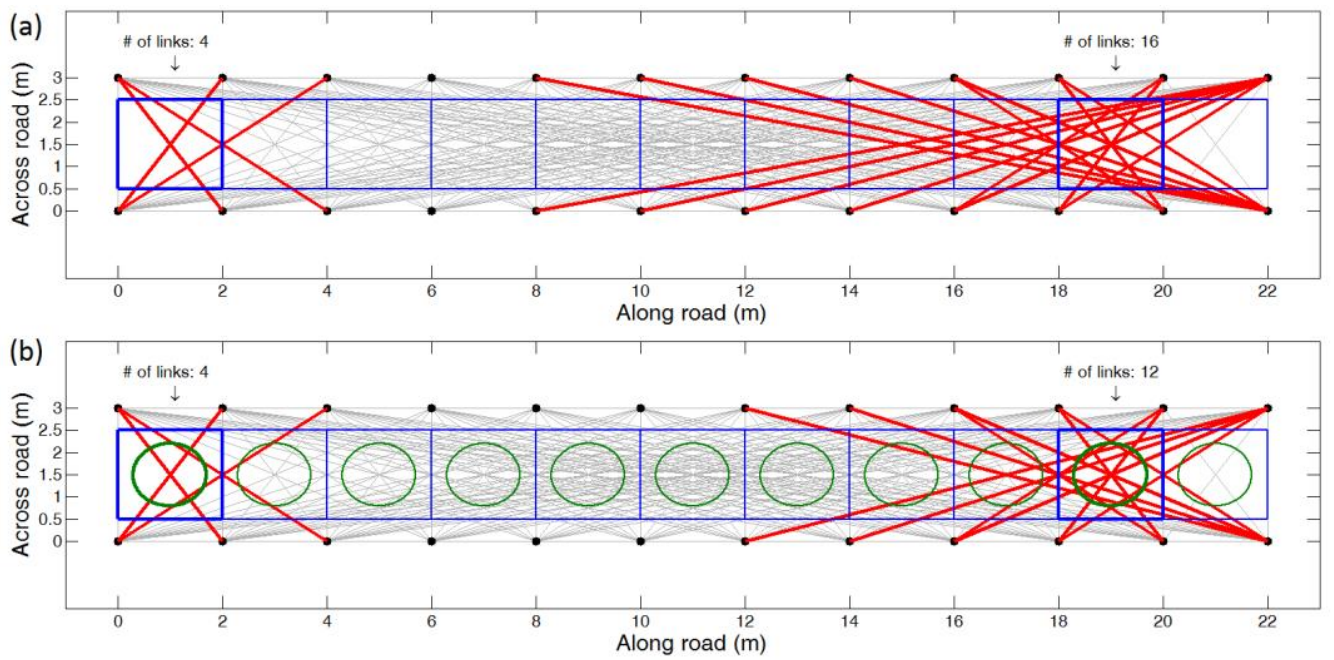

Fig. 9: Comparing which links and the amount of links are selected for pixels 1 and 10 using the (a) original and (b) newly proposed weight matrix calculation (WMC) method. The red and gray lines are the selected and unselected links respectively, the blue squares are the pixels and the selection circles with radius of $0.7 \mathrm{~m}$ are in green.

The performance results indicate that on most pixels, a higher accuracy of between $90 \%$ and $100 \%$ is achieved between pixels 1 and 9 using the newly proposed weight matrix calculation (WMC) method. This already demonstrates that the proposed RTI system will work near perfect according to the simulation model. Especially a higher accuracy of approximately $100 \%$ is yielded on pixels 7 and 8 , which are close to the center of the network, due to invaluable links being left out. The edge of the network shows similar performance between the methods of around $65 \%$ to $70 \%$, because in both cases only 4 links were selected. Pixels 9 and 10 give worst performance, because the pixel intensity 
on pixel 11 is close to the threshold value. When it is higher than the threshold, the system detected the vehicle location on pixel 11 instead of pixel 10, thereby introducing a False Positive (FP).

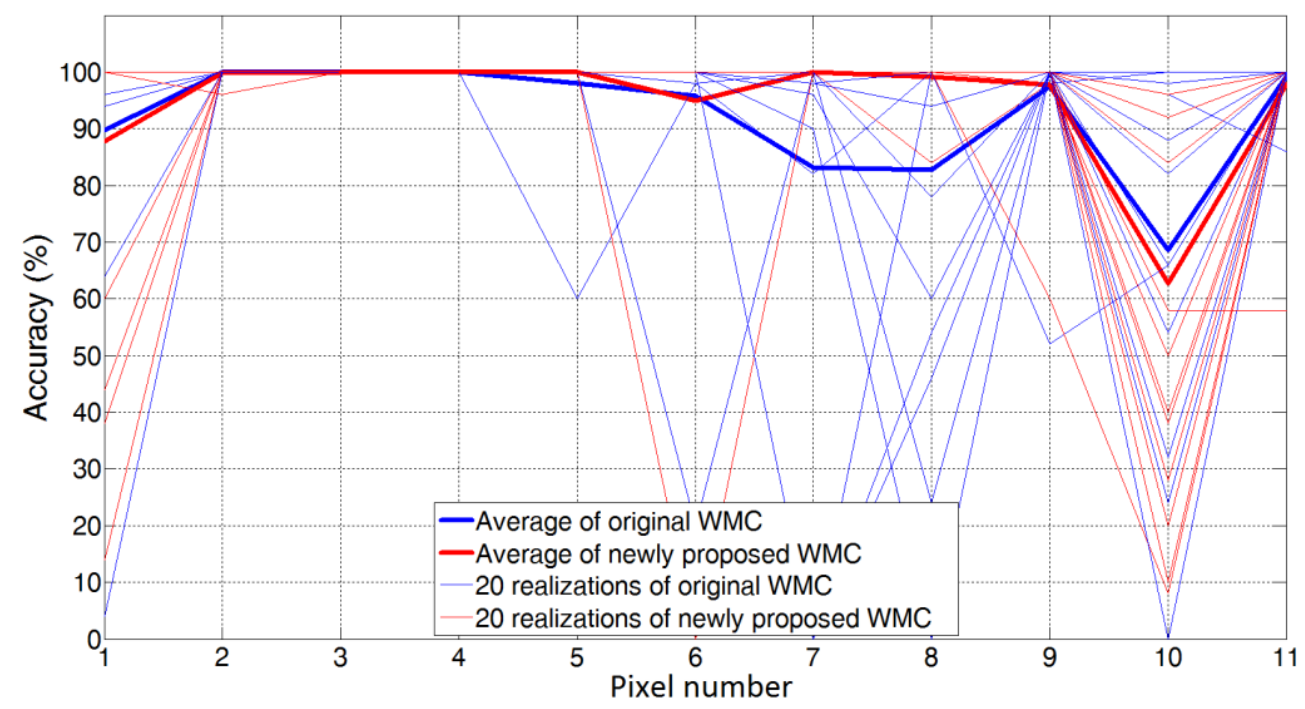

Fig. 10: Accuracy (\%) of vehicle detection on pixels 1 to 11 with original and new matrix calculation. The data presented are 20 realizations of the original and newly proposed weight matrix calculation (WMC) method and their average.

\section{2) Effect of node placement}

The second newly proposed technique is the non-linear spacing between nodes to increase link density at the network edges. A higher link density in a given area means more information is available about that area, theoretically leading to improved accuracy. Fig. 11 illustrates the number of selected links for pixels 1 and 10 using evenly and unevenly spaced nodes.
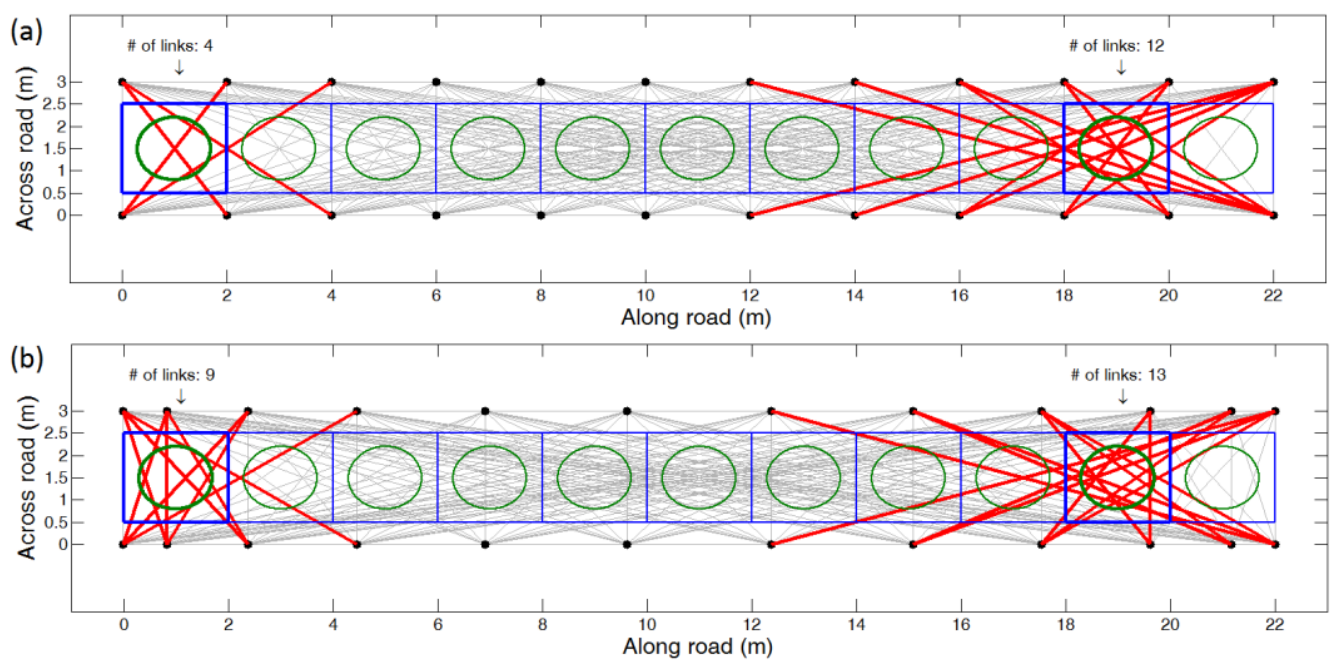

Fig. 11: Comparing which links and the amount of links are selected for pixels 1 and 10 using (a) evenly and (b) unevenly spaced nodes. The red and gray lines are the selected and unselected links respectively, the blue squares are the pixels and the selection circles with radius of $0.7 \mathrm{~m}$ are in green.

As shown, the number of selected links is more than doubled at the outer pixels (pixels 1 and 11, where 11 is symmetrical to 1), from 4 to 9 links. This comes at the cost of less reliable links in the center due to increased node spacing, but should theoretically not cause significantly reduced performance. 
The effect of node spacing on system performance is illustrated in Fig. 12, which was calculated using the same performance measuring procedure as in subsection IV.B.1). As can be observed, using unevenly spaced nodes leads to higher vehicle detection accuracy, especially on the network edges. This follows exactly the theory of how link density affects performance. System accuracy now is roughly speaking the same in the edges as in the center of the network, yielding a near perfect accuracy of between $95-100 \%$ in the performed experiments.

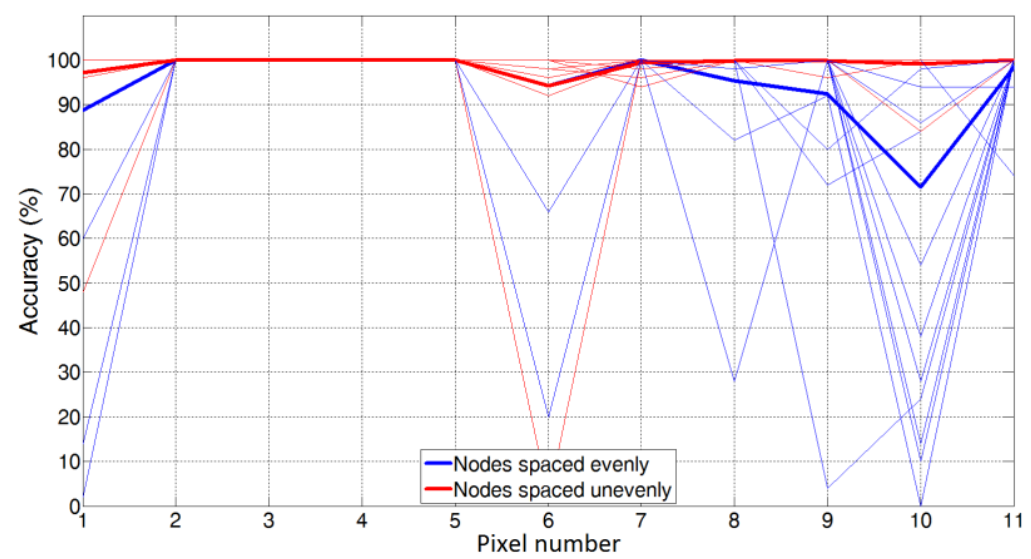

Fig. 12: Accuracy (\%) of vehicle detection on pixels 1 to 11 with evenly and unevenly spaced nodes. The data presented are 20 realizations of the evenly and unevenly spaced nodes (thinner blue and red curves respectively) and their average.

3) Effect of link-channel selection method

According to literature, the more frequency channels that are employed, the higher the quality (with least RSS variance) of link-channel pairs that are selected.

In the performed simulation experiments shown in Fig. 13, the RTI system demonstrates to be accurate using only one frequency channel without link-channel pair selection (LCPS), with an accuracy of between $80 \%$ and $90 \%$ for all pixels. However, adding a second channel and using the LCPS method, accuracy for all pixels is significantly improved to between $95 \%$ and $100 \%$. A perfect performance of $100 \%$ for all pixels is achieved using four channels and the LCPS method. It is likely that using three channels would also yield perfect performance, but was not tested for this simulation comparison. The performance result seen in Fig. 13 can be regarded as another type of simulation model validation, because the demonstrated performance difference between number of channels used confirms what is being described in literature. 


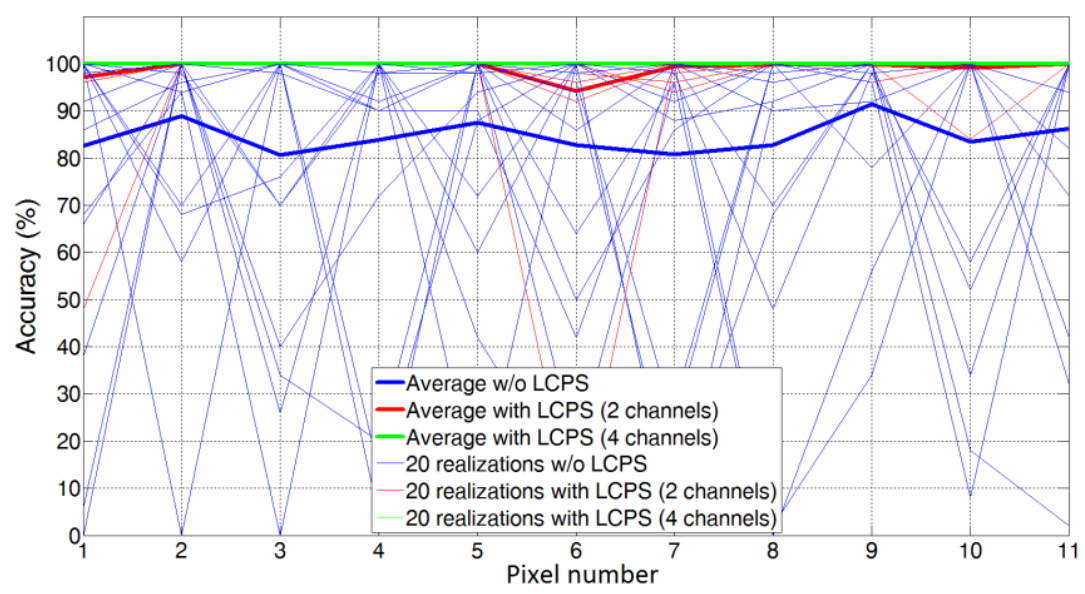

Fig. 13: Accuracy (\%) of vehicle detection on pixels 1 to 11 without link-channel pair selection (LCPS) and with LCPS (2 and 4 channels). The data presented are 20 realizations of the concerning variables and their average.

The proposed RTI system contains the use of two frequency channels, meaning that in theory, it performs close to perfect and almost as well as using more than two channels.

Fig. 14 illustrates two RTI images that compare the result of using two and four channels respectively, with their line plots displayed accordingly. The line plot also shows the result of using only one channel without LCPS. As expected, the curves with LCPS are very similar to each other due to their similar system performance, while the curve without LCPS is visibly different. Not only is the RTI curve different, but also its threshold, which is irregular and seemingly more random as opposed to the other curved and smooth thresholds. This irregular threshold is likely to have been the cause of the reduced vehicle detection accuracy. The reason for the irregular threshold is that all fade levels are included in the calculation, both positive and negative.

(a)

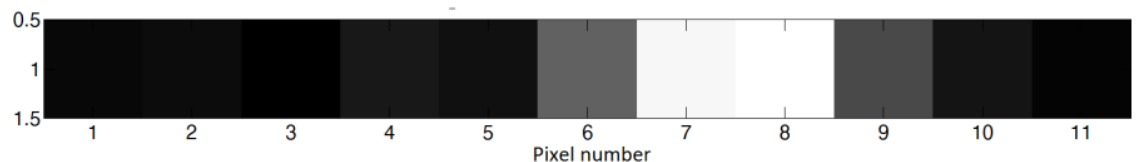

(b)

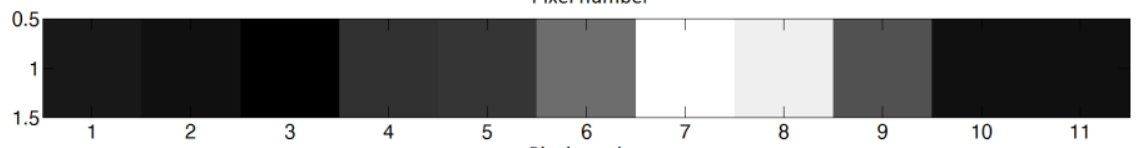

(c)

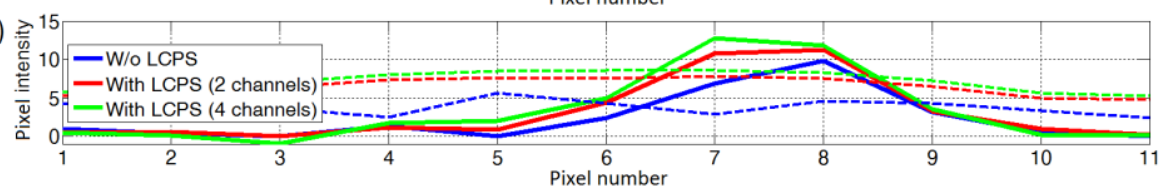

Fig. 14: RTI image with vehicle located on pixels 7 and 8 and corresponding line plot without link-channel pair selection (LCPS) and with LCPS using (a) 2 and (b) 4 channels. The dashed curves in (c) are the thresholds with their color corresponding to the RTI image they represent.

4) Parameters used for optimized result

The parameters for the threshold $\rho, n$ and $\alpha$ were optimized empirically, by trying out different values and observing which values yield the best vehicle detection performance. Note that the optimized values may not be the true best, but merely an approximation, as it is a cumbersome task to optimize parameters manually. Table 2 summarizes the parameter values used to obtain the optimized result. 
Journal of Microwaves, Optoelectronics and Electromagnetic Applications, Vol. 15, No. 4, December 2016495 DOI: http://dx.doi.org/10.1590/2179-10742016v15i4648

Table 2: Parameters and their corresponding values and description used for the optimized result.

\begin{tabular}{ccc}
\hline Parameter & Value & Description \\
\hline$\rho$ & 2 & Threshold weighting parameter \\
$n$ & 4 & Threshold power parameter \\
$r$ & 0.7 & Link selection circle radius \\
$\alpha$ & 0.1 & Generalized regularization parameter \\
\hline
\end{tabular}

\section{CONCLUSION}

This paper proposes a new simulation model that allows the simulation of RSS data in arbitrary RTI networks. The simulation model was validated by comparing the Burr distribution approximation of the RSS histograms of the experimental data offered by Wilson et al. [7] and optimized simulated data. The simulation model was optimized by setting values of three path-loss model parameters that minimize the MSE between the simulated and reference histograms. An implementation of the simulation model is presented, which simulates an enhancement of a previously proposed RTI system by Wilkens et al. [6]. The enhancement includes three newly implemented techniques, namely a new weight matrix calculation method, a new node spacing setup and a new pixel-specific threshold calculation approach used for car detection, localization and speed estimation. Simulation results presented in section IV have demonstrated that the two former newly introduced methods improved system performance considerably compared to current approaches. The results of the performed simulated experiments indicate that it is possible to detect both one and two family sized cars simultaneously in the proposed RTI network setup. Vehicle detection performance is demonstrated to be between $95 \%$ and $100 \%$, which is near perfect, using the new WMC method, new node spacing setup and using two frequency channels for the link-channel pair selection method. The desired outcome of this paper is the continuation of the presented research, eventually hoping to deploy the RTI network as a (complementary) vehicle traffic monitoring structure integrated into an Intelligent Transport System.

One of the most significant future research is the practical implementation of the proposed RTI system. To improve simulation model "realisticness", future research may involve developing a more sophisticated signal attenuation model. Furthermore, a more advanced technique for vehicle detection may be implemented using digital image processing techniques. The proposed method of thresholding is relatively simple and may not perform as well in a practical situation as it did in the simulation model. Additionally, to minimize RTI system costs, the minimum amount of sensors required to obtain a given system performance level may be investigated. It would also be interesting to apply optimization techniques that search for the optimized parameter values automatically.

\section{REFERENCES}

[1] N. Patwari and P. Agrawal, "Effects of Correlated Shadowing: Connectivity, Localization, and RF Tomography," Information Processing in Sensor Networks, IPSN 2008, pp. 82-93. IEEE, 2008.

[2] M. Bocca, O. Kaltiokallio and N. Patwari, "Radio Tomographic Imaging for Ambient Assisted Living," Evaluating AAL Systems Through Competitive Benchmarking, pp. 108-130. Springer, 2013.

[3] C. Alippi, M. Bocca, G. Boracchi, N. Patwari and M. Roveri, "RTI Goes Wild: Radio Tomographic Imaging for Outdoor People Detection and Localization," IEEE, 2014. 
Journal of Microwaves, Optoelectronics and Electromagnetic Applications, Vol. 15, No. 4, December 2016496 DOI: http://dx.doi.org/10.1590/2179-10742016v15i4648

[4] C. Cooke, "Attenuation Field Estimation Using Radio Tomography," Faculty of the Virginia Polytechnic Institute and State University, Blacksburg, 2011.

[5] A. Milburn, "Algorithms and models for radio tomographic imaging," Leiden University, Leiden, 2014.

[6] J. T. Wilkens and A. S. Garcia, "Design for Multi-Vehicle Roadside Tracking Based on Radio Tomographic Imaging," Anais Completo da Programação Técnica. XXXIII Simpósio Brasileiro de Telecomunicações, 2015.

[7] J. Wilson and N. Patwari, "Radio Tomographic Imaging Data Set," Sensing and Processing Across Networks at Utah, 21 Jan 2010. [Online]. Available: http://span.ece.utah.edu/rti-data-set. [Accessed 4 April 2016].

[8] J. Wilson and N. Patwari, "Radio Tomographic Imaging with Wireless Networks," IEEE Transactions on Mobile Computing, vol. 9, no. 5, pp. 621-632, 2010.

[9] Texas Instruments, "A USB-Enabled System-On-Chip Solution for 2.4-GHz IEEE 802.15.4 and ZigBee Applications," Texas Instruments, June 2010. [Online]. Available: http://www.ti.com/lit/ds/symlink/cc2531.pdf. [Accessed 4 April 2016].

[10] IEEE Computer Society, "Part 15.4: Wireless Medium Access Control (MAC) and Physical Layer (PHY) Specifications for Low-Rate Wireless Personal Area Networks (WPANs)," The Institute of Electrical and Electronics Engineers, Inc., New York, 2006.

[11] T. S. Rappaport, "Wireless Communications: Principles and Practice. Second Edition," Prentice Hall, 2002, pp. 6978, 102-104, 174-176.

[12] MathWorks, "Fading Channels," MathWorks, 2016. [Online]. Available: http://www.mathworks.com/help/comm/ug/fading-channels.html. [Accessed 4 April 2016].

[13] C. R. Anderson, R. K. Martin, O. T. Walker and R. T. Thomas, "Radio Tomography for Roadside Surveillance," IEEE Journal on Selected Topics in Signal Processing, vol. 8, no. 1, pp. 66-79, 2014.

[14] National Instruments, "Pulse-Shape Filtering in Communications Systems," 5 Nov 2014. [Online]. Available: http://www.ni.com/white-paper/3876/en/. [Accessed 4 April 2016]. 